\title{
Study of the Brittle Fracture of Monocrystalline Silicon Wafers
}

\author{
J. Barredo $\uparrow$, A. Fraileł and E. Alarcónł \\ $\uparrow$ Centre for Modelling in Mechanical Engineering (F2I2-CEMIM) \\ Department of Structural Mechanics and Industrial Constructions \\ Polytechnical University of Madrid, Spain
}

\begin{abstract}
There is a growing trend towards using thinner wafers in order to reduce the costs of solar energy. But the current tools employed during the solar cells production are not prepared to work with thinner wafers, decreasing the industrial yield due to the high number of wafers broken. To develop new tools, or modify existing ones, the mechanical properties have to be determined. This paper tackles an experimental study of the mechanical properties of wafers. First, the material characteristics are detailed and the process to obtain wafers is presented. Then, the complete test setup and the mechanical strength results interpreted by a described numerical model are shown
\end{abstract}

Keywords: four line bending test, finite elements, large displacement, monocrystalline silicon wafer, cleavage plane, size effect.

\section{Introduction}

Due to ecological problems caused by fossil fuel and in order to avoid energy shortages, it is key the development of clean energies. In this context, photovoltaic solar energy arises as a reliable alternative. The dominant issue of the photovoltaic industry is to fabricate solar cells in large volume that are both highly efficient and cost effective. The main absorber material, used today for the majority of commercially produced solar cells, is Czochralski grown crystalline silicon in monocrystalline form. The problem is that silicon wafer attributes to sometimes more than $50 \%$ of the module cost [1], [2]. One way to meet today's demand of lower wafer cost is employing thinner wafers. However, the handling of these during the production process without the modification of the tools involved, leads to an increase of broken wafers. So as a first step to develop new, or modify existing tools, the mechanical properties have to be determined. 
This paper shows the results of an experimental study of the mechanical properties of monocrystalline silicon wafers. To this end, several dozens of tests have been carried out to minimize the statistical error. The test description and the post process applied to results are explained in detail. Moreover, it has been necessary a complete study of the wafers properties to know characteristics that can influence the rupture process. These properties are summarized in the next point.

\section{Material description}

As it has been mentioned before, solar cells made out of Czochralski $(\mathrm{Cz})$ grown crystals and wafers play the dominant part in today's photovoltaic industry. The growth process starts when feedstock material, i.e. polycrystalline silicon pieces or residues from single crystals, is melted in a crucible and a seed crystal is first dipped into the melt. Then the seed is slowly withdrawn vertically to the melt surface whereby the liquid crystallises at the seed (figure 1).



Figure 1: Ingot grown by Czochralski

The ingot is pulled in a defined $\langle 100\rangle$ orientation. This is a big economic advantage since the solar cell process can use this crystallographic property to homogeneously texture solar cells with a very cost effective wet chemical etching step. By anisotropic etching a surface structure with random pyramids is built that couples the incoming light very effectively into the solar cell. Moreover, silicon grown in a monocrystalline form presents cleavage planes which are the crystallographic preferred fracture planes ([7], [8]). Their influence in the mechanical strength is studied in this paper.

On the other hand, it's known [1] that square cells are best suited to build a highly efficient solar module whereas $\mathrm{Cz}$ ingots have a round cross section. In order to use both the crystal and the module area best, the ingots are usually cut into pseudo- 
square cross section before they are cut into wafers. Standard sizes are about 10x10 $\mathrm{cm}^{2}$.

After crystal growth the silicon ingots are cut in a first step by band saws into columns that are glued to a substrate holder. Then, the silicon column on the holder is pushed against the moving wire web and sliced into hundreds of wafers at the same time (figure 2).

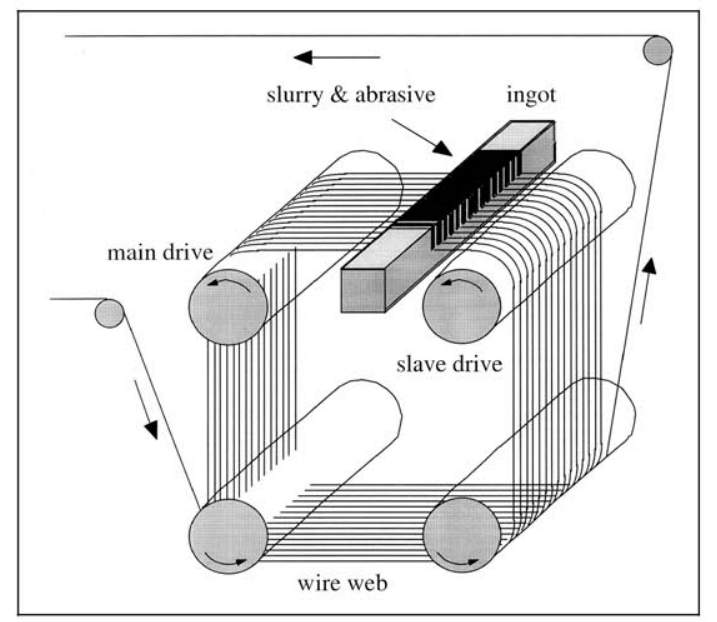

Figure 2: Multi-wire sawing technique

In this study, after cut by sawing, wafers thickness was decreased by means of a caustic soda bath. In this way, wafers of approximately 300, 200 and 100 microns thickness were obtained through different long baths.

\section{Test description}

In order to be conservative taking into account edge and surface defects, the four line bending test has been chosen to carry out the experimental study ([7]). To this end a testing machine has been acquired. Like is shown in figure 3, tests results are load-displacement curves:
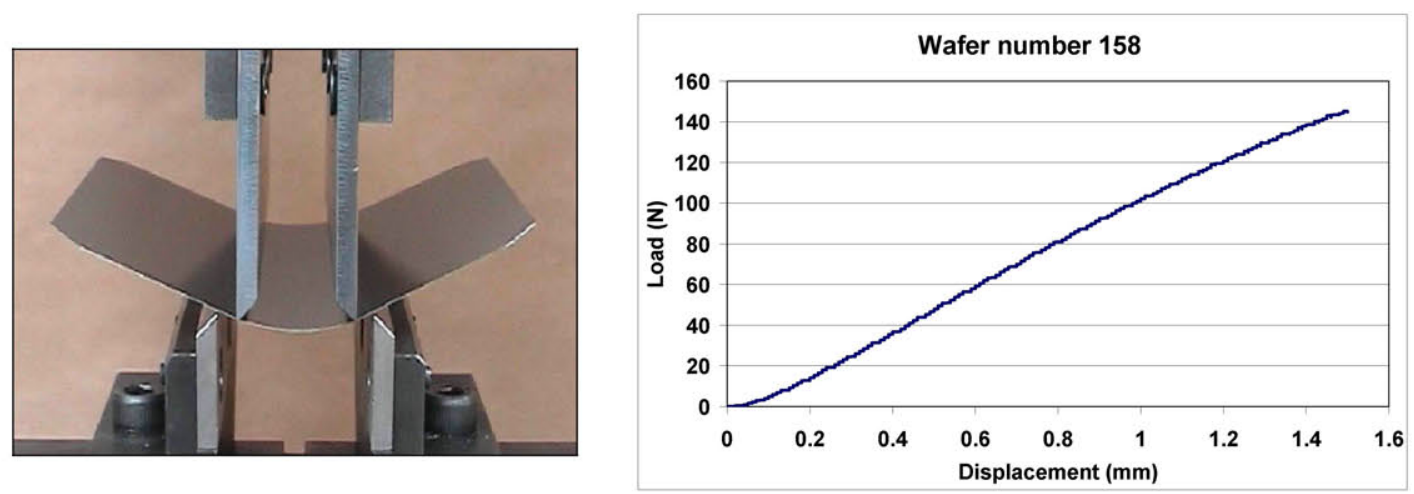

Figure 3: Photo and the resulting load-displacement curve 
Moreover tests have been recorded to study rupture process. Due to high crack propagation velocity a high speed camera has been employed recording videos at 1000 frames per second. In photo of figure 4 is shown the complete test setup.

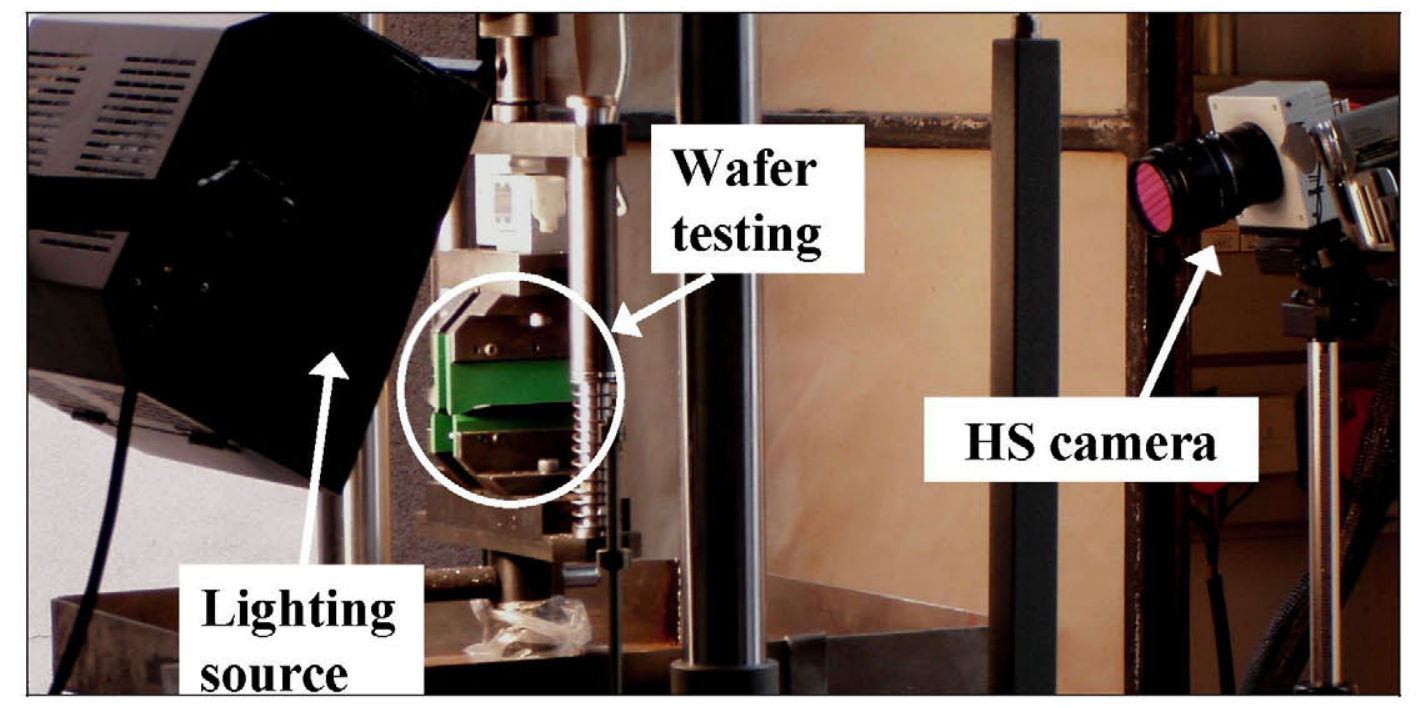

Figure 4: Test setup

Analysing the test videos, the rupture propagation through cleavage planes appears clearly. In figure 5 and 6 the wafers failure with different orientation of cleavage plane is shown.



Figure 5: Breakage of wafer with cleavage planes parallel to the edges 


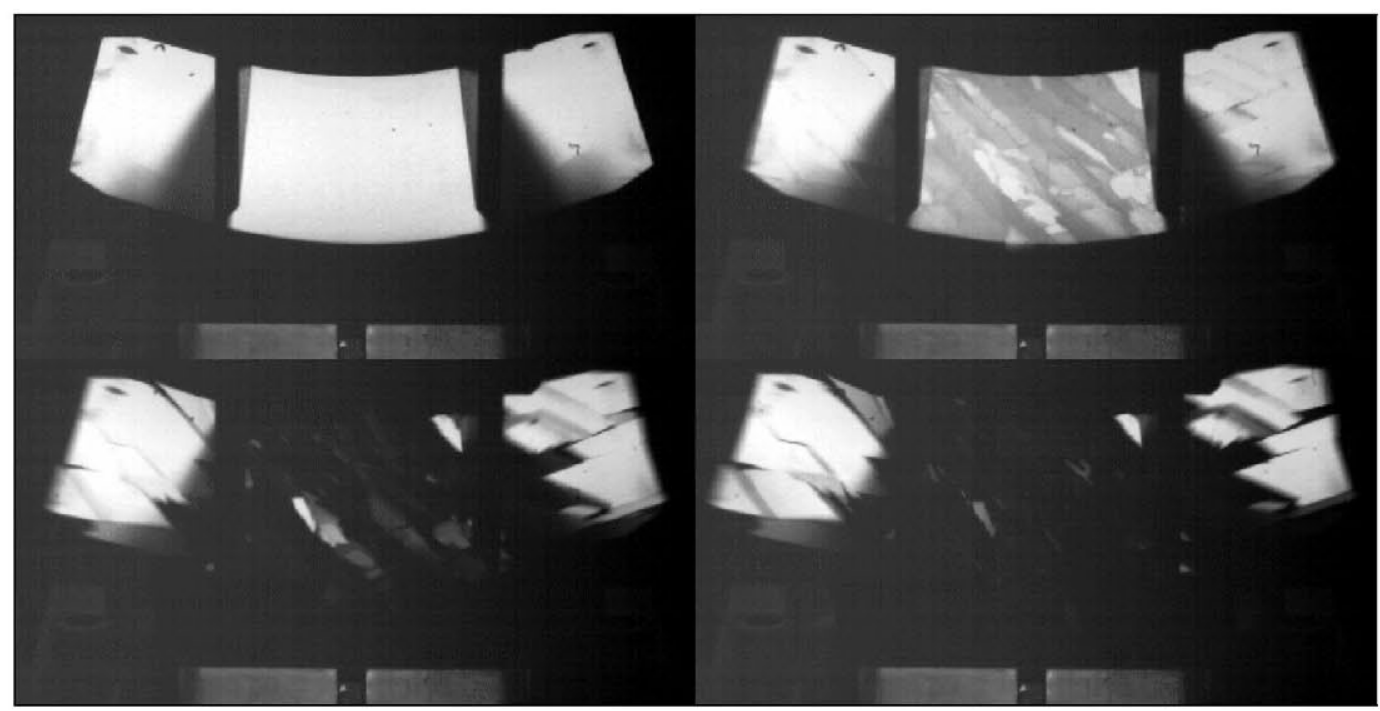

Figure 6: Breakage of wafer with cleavage planes with an angle to the edges

\section{Numerical model}

A numerical model is necessary to infer the mechanical properties from the tests. The model has to represent a non-linear behaviour due to two different factors.

- Large displacements.

- Sliding and friction between wafer and supports.

The Finite Element models have been set up with commercial software packages ADINA and ANSYS, employing shell and beam elements as well as different type of contact elements in combination with large displacement formulation. The model has been calibrated with test results (figure 7).

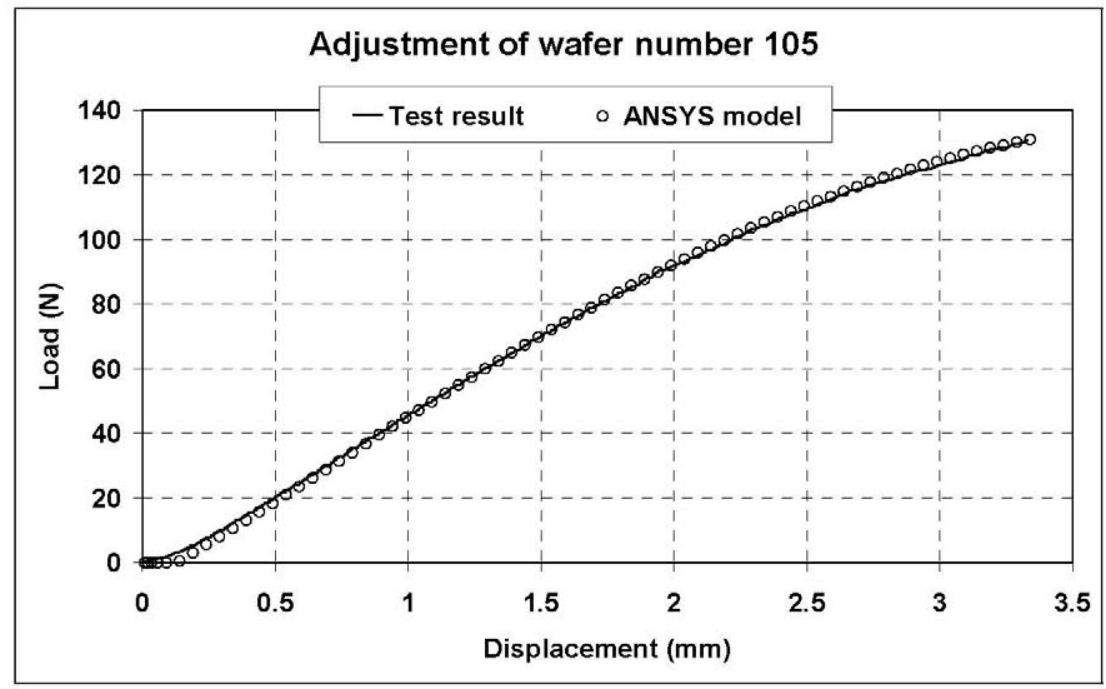

Figure 7: Adjustment of numerical model from test result 
A comparison between the results from the use of a simplified model or finite element model is shown in figure 8 and figure 9. The simplified model (figure 8 ) is clearly not sufficient.

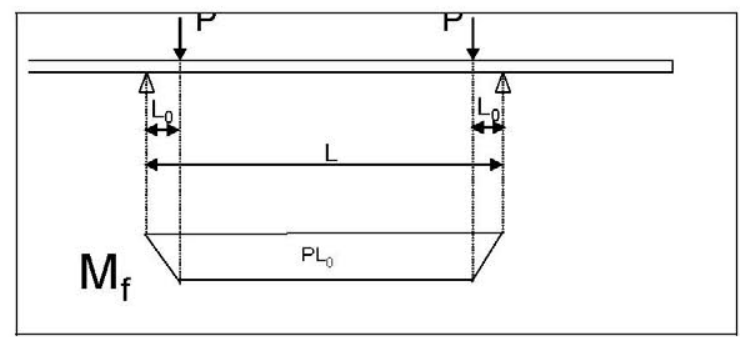

Wafer number 105

$$
\begin{aligned}
& \mathbf{P}_{\text {Total }}=2 \mathbf{P}=130.7 \mathrm{~N} \\
& \mathrm{~L}=0.06 \mathrm{~m} \\
& \mathrm{~L}_{0}=0.006 \mathrm{~m} \\
& \mathrm{~h}=275 \mu \mathrm{m} \text { (thickness) } \\
& \mathrm{b}=0.103 \mathrm{~m} \text { (depth) }
\end{aligned}
$$

Figure 8: Simplified model sketch

The maximum stress in this model is:

$$
\sigma_{\max }=\frac{\mathrm{M}_{\mathrm{f}}}{\mathrm{W}}=\frac{6 \cdot \mathrm{M}_{\mathrm{f}}}{\mathrm{b} \cdot \mathrm{h}^{2}}=302 \mathrm{MPa}
$$

While the same test for ANSYS model presents the next stress distribution:

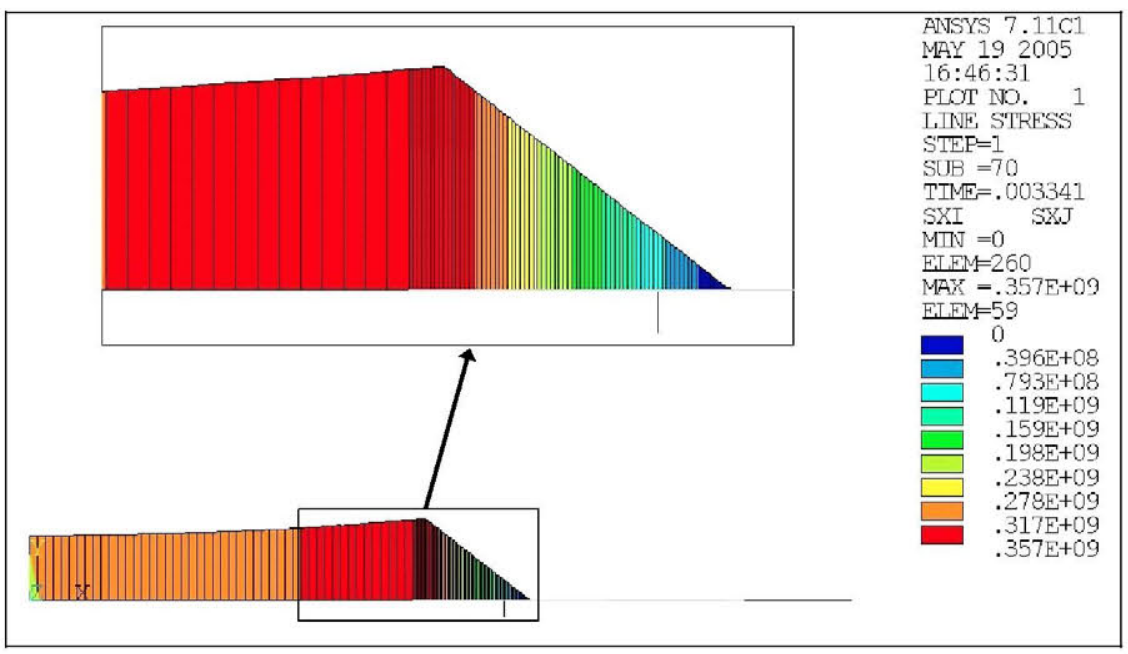

Figure 9: Stress distribution in finite element model for the example

As can be seen, stresses are not constant between loading application points and the maximum stress is $\sigma_{\max }=357 \mathrm{MPa}, 18 \%$ higher than the previous value.

\section{$5 \quad$ Test results}

The experimental study requires a statistical treatment of the test results to get a correct interpretation. As usual in brittle materials, Weibull statistics ([4], [6], [7], [8]) is employed in this case. 
Also, it's necessary to define a failure criterion studying the rupture mechanism. The failure occurs where the combination of stress and defect is critical ([4], [5]). Wafers present surface cracks caused by the cutting process ([1]) which influences the mechanical strength. Under loading, stress concentration at the crack tips can be sufficient to separate the bonds in cleavage planes and produce cracks propagation in cleavage plane directions. So, the influence of cleavage plane orientation in mechanical strength has been studied.

Several dozens of tests have been carried out. Result analysis starts with a correction due to size effect.

\subsection{Size effect}

In order to reduce the non-linear behaviour the distances between supports and between load application points have been modified for each wafer thickness.

Different distances lead to areas of constant stress of different sizes. So, due to size effect, higher bending strength and bigger scattered results are observed in thinner wafers (figure 10).

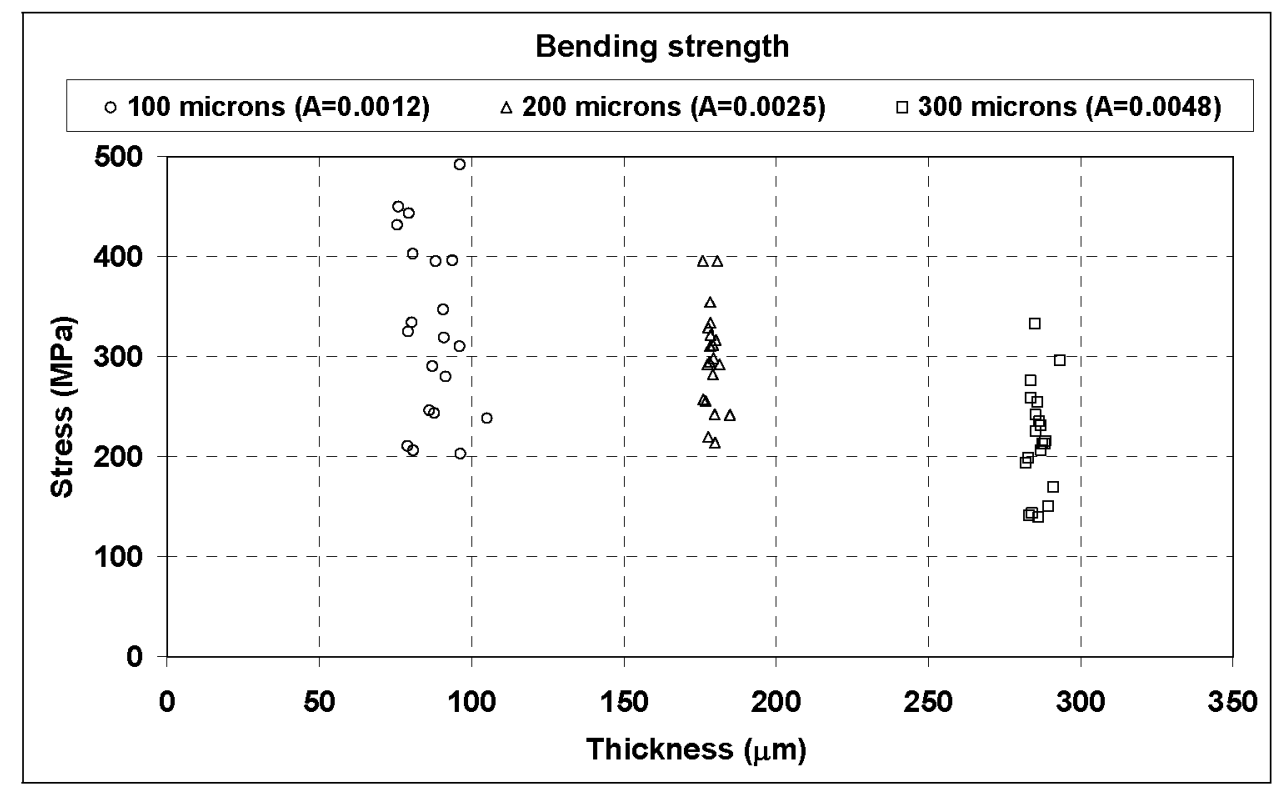

Figure 10: Bending strength versus wafer thickness

To compare test results, it's necessary to correct the size effect ([3], [4], [5], [6]). First of all, three Weibull triparameter distributions (figure 11) have been adjusted to fit the test results. Bending strength is adjusted:

$$
F(\sigma)=1-\exp \left[-\left(\frac{\sigma-N_{0}}{N_{a}-N_{0}}\right)^{\beta}\right]
$$


Where $\sigma$ is the bending strength, obtained by the numerical model, and $N_{0}, N_{a}$ and $\beta$ are Weibull parameters.

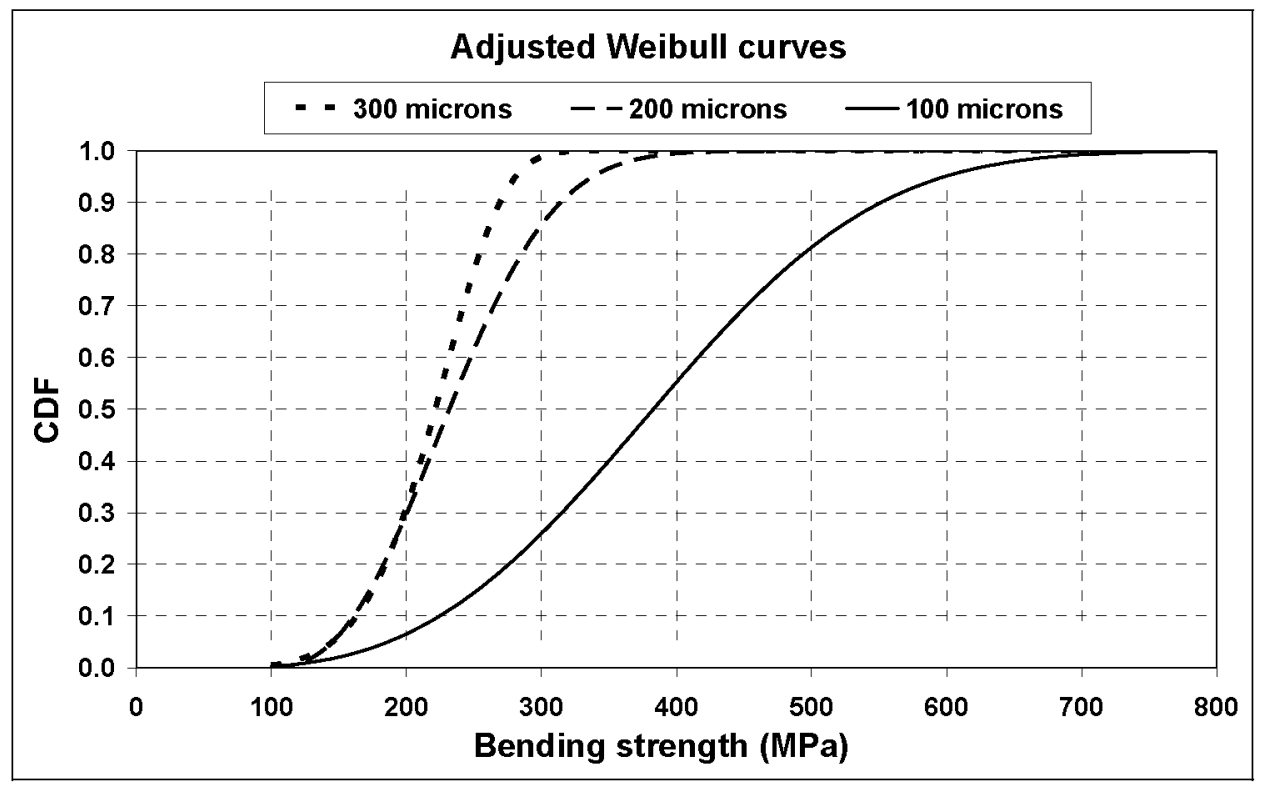

Figure 11: Adjusted Weibull curves

Next, reference area of each test is calculated. In the case of a four line bending test, reference area is a combination of the area of constant stress and the area of varying stress. Canteli et al. [4], [6] uses the expression:

$$
A_{\text {ref }}=b \cdot\left(L-2 L_{0}\right)+\frac{2 \cdot b \cdot L_{0}}{\beta+1}\left(1-\frac{N_{0}}{\sigma}\right)
$$

Where $b$ is wafer depth, $\mathrm{L}$ and $\mathrm{L}_{0}$ are test dimensions, $\sigma$ is the bending strength, and $\mathrm{N}_{0}$ and $\beta$ are Weibull parameters of the previous adjusted curve.

The reference area appears in the failure function through the reliability [3], [4]:

$$
\mathrm{R}(\sigma)_{\mathrm{A} 1}=\left[\mathrm{R}(\sigma)_{\mathrm{A} 2}\right]^{\mathrm{A} 1 / \mathrm{A} 2}
$$

In this way, the failure probability referred to a general area $\left(A_{\text {gen }}\right)$ from a particular area $\left(\mathrm{A}_{\text {part }}\right)$ is:

$$
F(\sigma)_{A_{\text {gen }}}=1-\exp \left[-\frac{A_{\text {gen }}}{A_{\text {part }}}\left(\frac{\sigma-N_{0}}{N_{a}-N_{0}}\right)^{\beta}\right]
$$

This expression modifies the probability associated to each bending strength result. Then, a new curve is fitted but in this case using the results obtained from the three 
values of thickness. The new adjustment implies some new distribution parameters which are obtained by an iterative process. Finally, in spite of testing wafers of different thicknesses with different tests dimensions, the convergence is achieved and a single failure probability curve (figure 12) represents the wafer strength.

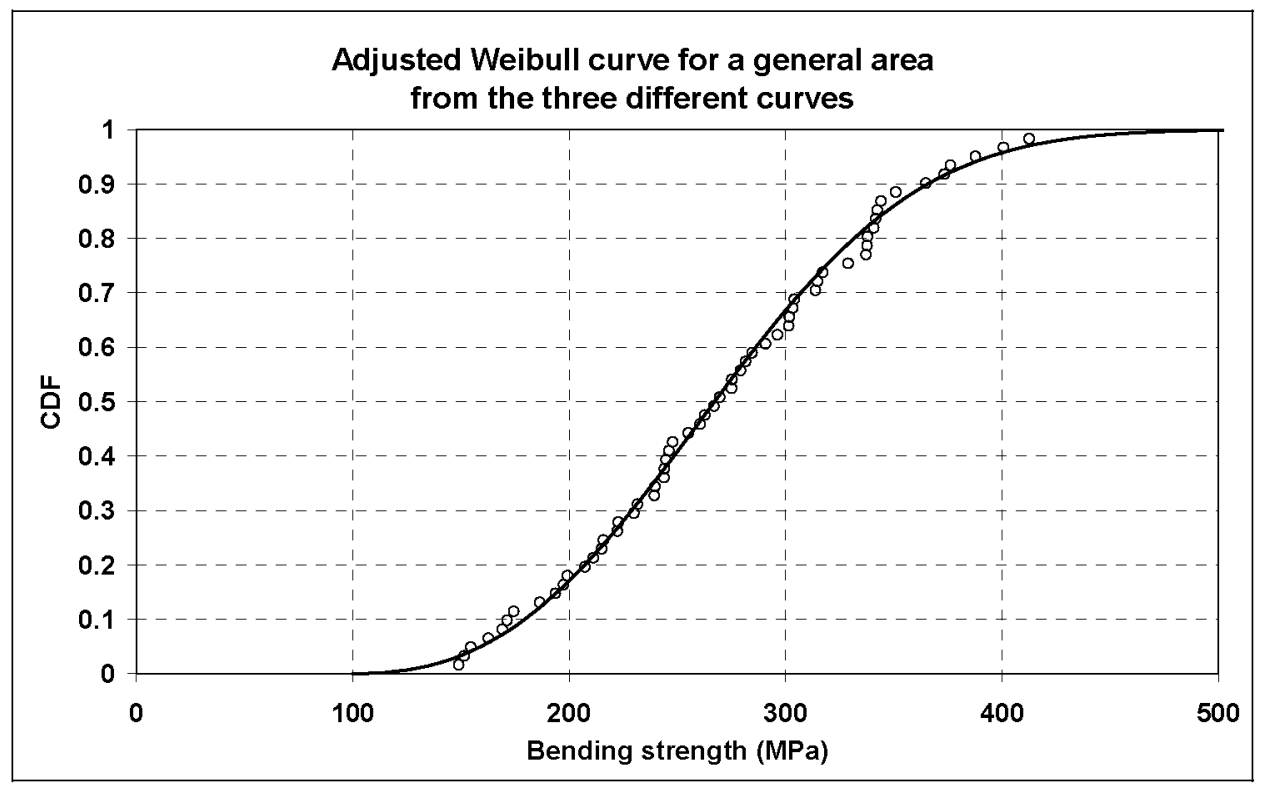

Figure 12: An only adjusted curve for all thicknesses

\subsection{Cleavage planes influence}

The curve obtained above represents the bending strength adjustment of commercial wafers. In order to study the influence of cleavage plane in mechanical strength, two additional types of wafers with different cleavage plane orientation (figure 13) have been tested.
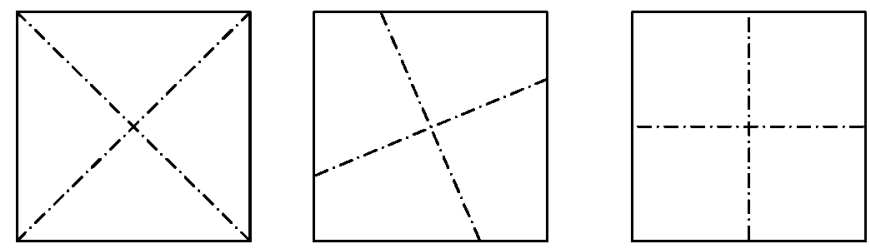

Figure 13: Sets of wafers prepared according to the orientation of cleavage planes

The first set of wafers, with diagonal cleavage planes, is the commercial one. The test results have been adjusted in the curve above (figure 12). The other sets have an angle of $22.5^{\circ}$ and $0^{\circ}$ to the wafer edge, respectively.

The study explained above is applied to the other two types of wafers, adjusting a curve for each one. Therefore, there are three different curves, one for each type of wafers, which must be referred to the same general area to allow comparison of the results (figure 14). 




Figure 13: Weibull adjustment for the three cleavage plane orientations

The results show that the curves are very close. In spite of the soda bath reduction of cracks and defects resulting in a better mechanical strength, the wafer failure is still determined by defects. The influence of cleavage planes is not as important as to justify a failure criterion based on them. Therefore, a general adjustment of all tests has been made, resulting in a failure curve of monocrystalline silicon wafers with good agreement with experimental results (figure 14). However, a relation between strength and cleavage plane orientation is observable and it's still being researched.

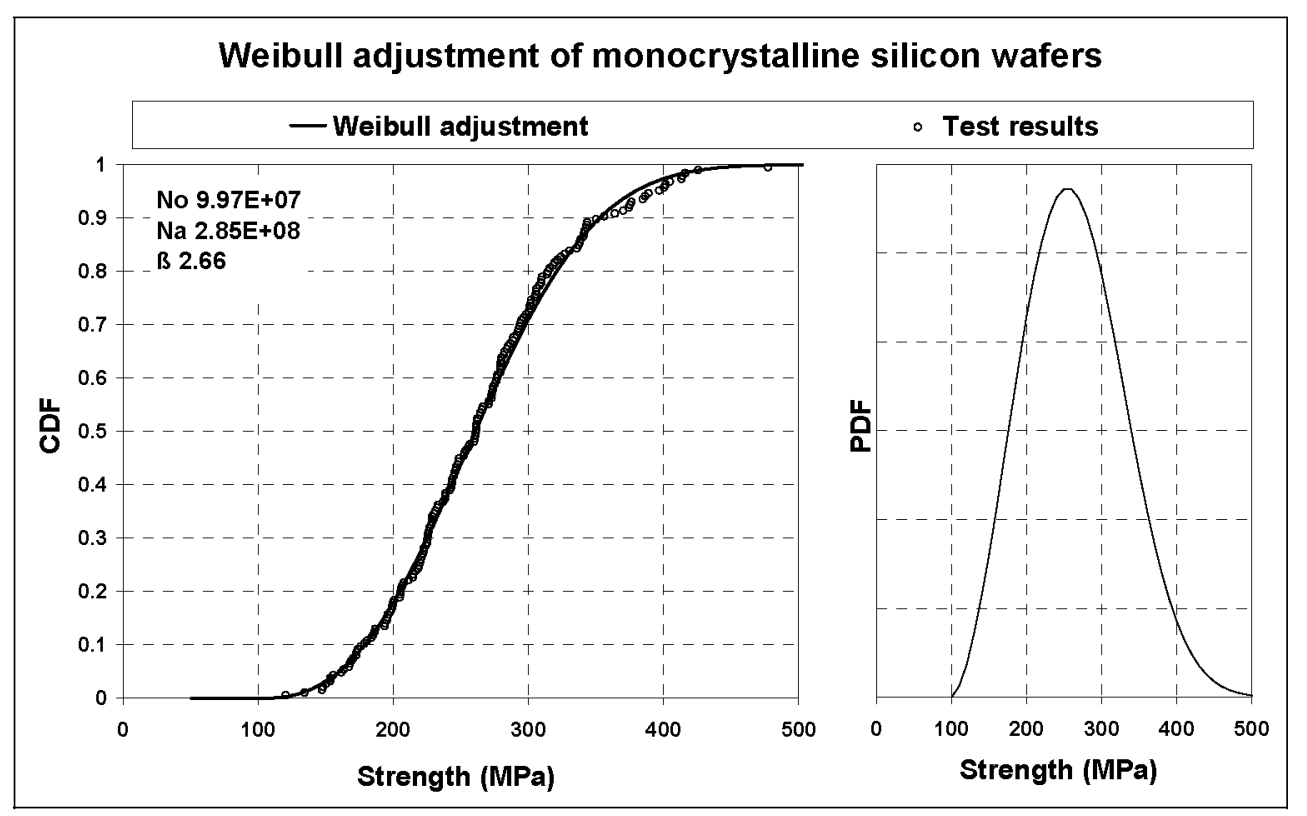

Figure 14: Weibull adjustment for test results 


\section{Conclusions}

The paper shows a detailed experimental study to determine mechanical properties of silicon wafers.

To characterize the breakage strength, a number of four line bending tests have been carried out. Tests have been recorded with a high speed camera, exhibiting rupture propagation through cleavage planes.

The influence of cleavage planes in the rupture process has been studied. To this end, three different types of wafer sets with different cleavage plane orientations have been prepared.

A post process is applied to the test results in order to take the size effect into account

According to the results, surface and edge cracks or defects determine the mechanical strength of wafers. The influence of cleavage plane orientations is not very important

The main result of the study is a unique failure curve with a very good correlation, valid for a wide range of thickness and cleavage plane orientations. This curve allows studying new service situations and can be used to develop new handling tools.

\section{Acknowledgement}

This work has been made under the sponsorship of the University and Investigation State Secretary, belonging to Spanish Ministry of Education and Science, in its contract TIC2002-04561-C03-01. The presented results have been obtained in cooperation with Instituto de Energía Solar (IES) and Teknologia Mikroelektronikoaren Institua (TIM). Authors want to thank Isofotón for providing us wafers to test.

\section{References}

[1] A. Luque, S. Hegedus, "Handbook of Photovoltaic Science and Engineering", John Wiley \& Sons Ltd, West Sussex, England, 2003.

[2] J. C. Jimeno, V. Rodríguez, R. Gutiérrez, F. Recart, G. Bueno, F. Hernando, "Very Low Thickness Monocrystalline Silicon Solar Cells", Sixteenth European Photovoltaic Solar Energy Conference, Glasgow, Scotland, 2000.

[3] W. Weibull, "A Statistical Theory of the Strength of Materials", Proceedings of the Royal Swedish Institute of Engineering Research Nr. 151, 1939

[4] M. A. García, "Probabilistic Design and Experimental Failure Analysis of Glass", PhD Thesis, Oviedo University, Spain, 2001

[5] D. Broek, "Elementary Engineering Fracture Mechanics", Martinus Nijhoff Publishers, 1986

[6] M.A. García, A. F. Canteli, M. J. Lamela, E. Castillo, "A Design Model for Glass Elements based on the Statistical Distribution of Crack Sizes", Eighth Conference Exhibition of the European Ceramic Society. Istanbul, Turkey, 2003. 
[7] B. Cotterell, Z. Chen, J. B. Han, N. X. Tan, "The Strength of the Silicon Die in Flip-Chip Assemblies", Journal of electronic packaging, ASME, USA, 2003.

[8] O. M. Jadaan, N. N. Nemeth, J. Bagdahn, W. N. Sharpe Jr, "Probabilistic Weibull Behaviour and Mechanical Properties of MEMS Brittle Materials", Journal of Material Science, 2003 